Advances in Intelligent Systems and Computing 290

Sigeru Omatu $\cdot$ Hugues Bersini

Juan M. Corchado · Sara Rodríguez

Paweł Pawlewski · Edgardo Bucciarelli Editors

\title{
Distributed Computing and Artificial Intelligence, 11th International Conference
}




\section{Advances in Intelligent Systems and Computing}

Volume 290

Series editor

Janusz Kacprzyk, Polish Academy of Sciences, Warsaw, Poland

e-mail: kacprzyk@ibspan.waw.pl

For further volumes:

http://www.springer.com/series/11156 


\section{About this Series}

The series "Advances in Intelligent Systems and Computing" contains publications on theory, applications, and design methods of Intelligent Systems and Intelligent Computing. Virtually all disciplines such as engineering, natural sciences, computer and information science, ICT, economics, business, e-commerce, environment, healthcare, life science are covered. The list of topics spans all the areas of modern intelligent systems and computing.

The publications within "Advances in Intelligent Systems and Computing" are primarily textbooks and proceedings of important conferences, symposia and congresses. They cover significant recent developments in the field, both of a foundational and applicable character. An important characteristic feature of the series is the short publication time and world-wide distribution. This permits a rapid and broad dissemination of research results.

\section{Advisory Board}

\section{Chairman}

Nikhil R. Pal, Indian Statistical Institute, Kolkata, India

e-mail: nikhil@isical.ac.in

Members

Rafael Bello, Universidad Central "Marta Abreu" de Las Villas, Santa Clara, Cuba e-mail: rbellop@uclv.edu.cu

Emilio S. Corchado, University of Salamanca, Salamanca, Spain

e-mail: escorchado@usal.es

Hani Hagras, University of Essex, Colchester, UK

e-mail: hani@essex.ac.uk

László T. Kóczy, Széchenyi István University, Győr, Hungary

e-mail: koczy@sze.hu

Vladik Kreinovich, University of Texas at El Paso, El Paso, USA

e-mail: vladik@utep.edu

Chin-Teng Lin, National Chiao Tung University, Hsinchu, Taiwan

e-mail: ctlin@mail.nctu.edu.tw

Jie Lu, University of Technology, Sydney, Australia

e-mail: Jie.Lu@uts.edu.au

Patricia Melin, Tijuana Institute of Technology, Tijuana, Mexico

e-mail: epmelin@hafsamx.org

Nadia Nedjah, State University of Rio de Janeiro, Rio de Janeiro, Brazil

e-mail: nadia@eng.uerj.br

Ngoc Thanh Nguyen, Wroclaw University of Technology, Wroclaw, Poland

e-mail: Ngoc-Thanh.Nguyen@pwr.edu.pl

Jun Wang, The Chinese University of Hong Kong, Shatin, Hong Kong

e-mail: jwang@mae.cuhk.edu.hk 
Sigeru Omatu · Hugues Bersini

Juan M. Corchado · Sara Rodríguez

Paweł Pawlewski · Edgardo Bucciarelli

Editors

\section{Distributed Computing and Artificial Intelligence, 11th International Conference}

囪 Springer 


\section{Editors}

Sigeru Omatu

Faculty of Engineering

Osaka Institute of Technology

Osaka

Japan

Hugues Bersini

Université Libre de Bruxelles

Brussels

Belgium

Juan M. Corchado

Faculty of Science

Department of Computing Science and Control

University of Salamanca

Salamanca

Spain

\author{
Sara Rodríguez \\ Faculty of Science \\ Department of Computing Science \\ and Control \\ University of Salamanca \\ Salamanca \\ Spain
}

Paweł Pawlewski

Faculty of Engineering Management

Poznan University of Technology

Poznan

Poland

Edgardo Bucciarelli

Dep. PPEQS, Section of Economics and Quantitative Methods

University of Chieti-Pescara

Pescara

Italy
ISSN 2194-5357

ISBN 978-3-319-07592-1

DOI 10.1007/978-3-319-07593-8

Springer Cham Heidelberg New York Dordrecht London
ISSN 2194-5365 (electronic) ISBN 978-3-319-07593-8 (eBook)

Library of Congress Control Number: 2014939945

(c) Springer International Publishing Switzerland 2014

This work is subject to copyright. All rights are reserved by the Publisher, whether the whole or part of the material is concerned, specifically the rights of translation, reprinting, reuse of illustrations, recitation, broadcasting, reproduction on microfilms or in any other physical way, and transmission or information storage and retrieval, electronic adaptation, computer software, or by similar or dissimilar methodology now known or hereafter developed. Exempted from this legal reservation are brief excerpts in connection with reviews or scholarly analysis or material supplied specifically for the purpose of being entered and executed on a computer system, for exclusive use by the purchaser of the work. Duplication of this publication or parts thereof is permitted only under the provisions of the Copyright Law of the Publisher's location, in its current version, and permission for use must always be obtained from Springer. Permissions for use may be obtained through RightsLink at the Copyright Clearance Center. Violations are liable to prosecution under the respective Copyright Law.

The use of general descriptive names, registered names, trademarks, service marks, etc. in this publication does not imply, even in the absence of a specific statement, that such names are exempt from the relevant protective laws and regulations and therefore free for general use.

While the advice and information in this book are believed to be true and accurate at the date of publication, neither the authors nor the editors nor the publisher can accept any legal responsibility for any errors or omissions that may be made. The publisher makes no warranty, express or implied, with respect to the material contained herein.

Printed on acid-free paper

Springer is part of Springer Science+Business Media (www.springer.com) 


\section{Contents}

\section{AI-Driven Methods for Multimodal Networks and Processes Modeling}

Reachability Modeling for Multimodal Networks Prototyping

Grzegorz Bocewicz, Robert Wójcik, Zbigniew Banaszak

Hybrid Solution Framework for Supply Chain Problems

Paweł Sitek, Jarosław Wikarek

Scheduling of Mobile Robots with Preemptive Tasks

Izabela Nielsen, Quang-Vinh Dang, Peter Nielsen, Pawel Pawlewski

Multimodal Processes Approach to Supply Chain Modeling

Patrycja Hoffa, Pawel Pawlewski, Izabela Nielsen

Multimodal Perspective on Ontologies Combining Problem in Production

Management Systems

Przemysław Różewski, Justyna Bednarz.

\section{Multi-Agents Macroeconomics}

Behavioral Macroeconomics and Agent-Based Macroeconomics

Shu-Heng Chen, Umberto Gostoli

Heterogeneous Households: Monopolistic Capitalists, Entrepreneurs and Employees

Jonathan Swarbrick

When Can Cognitive Agents Be Modeled Analytically versus

Computationally?

Leigh Caldwell 
Designing a Homo Psychologicus More Psychologicus: Empirical

Results on Value Perception in Support to a New Theoretical

Organizational-Economic Agent Based Model

Andrea Ceschi, Enrico Rubaltelli, Riccardo Sartori

Differences between Entrepreneurs and Managers in Large

Organizations: An Implementation of a Theoretical Multi-Agent Model

on Overconfidence Results

Riccardo Sartori, Andrea Ceschi, Andrea Scalco

The Empirical Microstructure of Agent-Based Models:

Recent Trends in the Interplay between ACE and Experimental

Economics

Paola D'Orazio, Marcello Silvestri

Households Debt Behavior and Financial Instability: Towards an

Agent-Based Model with Experimentally Estimated Behavioral Rules

Paola D'Orazio

Firm Size Distribution in Oblivious Equilibrium Model

with Quality Ladder . . . . . . . . . . . . . . . . . . . . .

Tetsushi Murao

Modeling Uncertainty in Banking Networks

Stojan Davidovic, Mirta Galesic, Konstantinos Katsikopoulos,

Nimalan Arinaminpathy

\section{Artificial Intelligence Applications}

Changing the Hidden Rules - An Excel Template for Discussing Soccer's Competitive Balance

Joaquim Teixeira, Nuno Santos, Paulo Mourao

Insider Trading, Earnings and Stock Based Compensation:

A View to Speculation

Esther B. Del Brio, Ilidio Lopes-e-Silva, Javier Perote

Service-Oriented Architectures: From Design to Production Exploiting

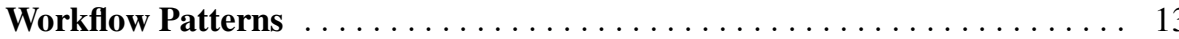

Maurizio Gabbrielli, Saverio Giallorenzo, Fabrizio Montesi

Reinforcement Learning Based on the Bayesian Theorem for Electricity

Markets Decision Support

Tiago M. Sousa, Tiago Pinto, Isabel Praça, Zita Vale, Hugo Morais

Distributed and Guided Genetic Algorithm for Humanitarian Relief

Planning in Disaster Case

Fethi Mguis, Kamel Zidi, Khaled Ghedira, Pierre Borne 
FleSe: A Tool for Posing Flexible and Expressive (Fuzzy) Queries to a

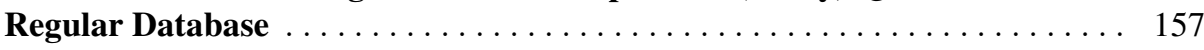

Víctor Pablos-Ceruelo, Susana Muñoz-Hernández.

Software Fault Prediction Based on Improved Fuzzy

Clustering . .

Golnoosh Abaei, Ali Selamat

Facial Authentication before and after Applying the Smowl Tool

in Moodle

Francisco D. Guillén-Gámez, Iván García-Magariño

SOA Modeling Based on MDA

Haeng-Kon Kim, Tai-Hoonn Kim

Intelligent Lighting Control System

Elena García, Sara Rodríguez, Juan F. De Paz, Javier Bajo

\section{Multi-Agent Systems}

Norm's Benefit Awareness in Open Normative Multi-agent Communities:

A Conceptual Framework

Al-Mutazbellah Khamees Itaiwi, Mohd Sharifuddin Ahmad,

Moamin A. Mahmoud, Alicia Y.C. Tang

The Geranium System: Multimodal Conversational Agents

for E-learning

David Griol, José Manuel Molina, Araceli Sanchis de Miguel

DiSEN-AlocaHR: A Multi-Agent Mechanism for Human Resources

Allocation in a Distributed Software Development Environment

Lucas O. Teixeira, Elisa H.M. Huzita

Multi-Agent Web Recommendations

Joaquim Neto, A. Jorge Morais

Designing Strategies for Improving the Performance of Groups in

Collective Environments .

L.F. Castillo, M.G. Bedia, C. Lopez, F.J. Seron, G. Isaza

Multiagent Application in Mobile Environments to Data Collection in

Park Zones

María Navarro, Fernando de la Prieta, Gabriel Villarrubia,

Mohd Saberi Mohamad

Organizational Architectures for Large-Scale Multi-Agent Systems'

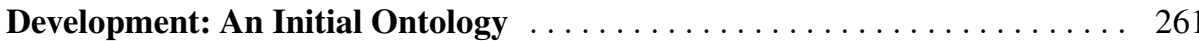

Markus Schatten 


\section{Distributed, Grid, Cloud and Mobile Computing}

Exploring the Role of Macroeconomic Mechanisms in Voluntary

Resource Provisioning in Community Network Clouds . . . . . . . . . . . . . . 269

Amin M. Khan, Felix Freitag

Performance and Results of the Triple Buffering Built-In in a Raspberry

PI to Optimize the Distribution of Information from a Smart Sensor . . . . . . 279

Jose-Luis Jimenez-Garcia, Jose-Luis Poza-Luján,

Juan-Luis Posadas-Yagüe, David Baselga-Masia,

José-Enrique Simó-Ten

Mobile Access to Sensor Network: A Use Case on Wildfire Monitoring

287

Sergio Trilles, Óscar Belmonte, Joaquín Huerta

Building Scalable View Module of Object-Oriented Database

Haeng-Kon Kim, Hyun Yeo

\section{Bioinformatics, Biomedical Systems, E-health}

E-Nose System by Using Neural Networks

Sigeru Omatu, Mitsuak Yano

Modelling an Orientation System Based on Speculative Computation

João Ramos, Ken Satoh, Paulo Novais, José Neves

Stable Learning for Neural Network Tomography by Using Back

Projected Image

Masaru Teranishi, Keita Oka, Masahiro Aramoto

Security Considerations for Patient Telemonitoring Schemes through

Wireless Networks

V. Morales, D. Cortés, N. Gordillo, A. De la Torre, D. Azpetia

Development of an Ontology for Supporting Diagnosis in Psychiatry

Cátia Silva, Goreti Marreiros, Nuno Silva

Augmented Reality Sign Language Teaching Model for Deaf Children . . . . 351

Jorge Jonathan Cadeñanes Garnica, María Angélica González Arrieta

A Multi-agent Simulation: The Case of Physical Activity and Childhood

Obesity

Rabia Aziza, Amel Borgi, Hayfa Zgaya, Benjamin Guinhouya

The Absorptive Capacity-Based View of Training: Enhancing Organizational Performance. An Exploratory Study in Spanish Family

Businesses

Felipe Hernández Perlines, María Yolanda Salinero Martín,

Benito Yáñez Araque 


\section{Data Mining, Information Extraction, Semantic, Knowledge Representation}

LIWC-Based Sentiment Analysis in Spanish Product Reviews

Estanislao López-López, María del Pilar Salas-Zárate,

Ángela Almela, Miguel Ángel Rodríguez-García, Rafael Valencia-García,

Giner Alor-Hernández.

Data Extraction Tool to Analyse, Transform and Store Real Data from

Electricity Markets

Ivo F. Pereira, Tiago M. Sousa, Isabel Praça, Ana Freitas,

Tiago Pinto, Zita Vale, Hugo Morais

Are There Semantic Primes in Formal Languages?

Johannes Fähndrich, Sebastian Ahrndt, Sahin Albayrak

The Age of Confidentiality: A Review of the Security in Social Networks and Internet .

Antonio Juan Sánchez, Yves Demazeau

Extracting Sentences Describing Biomolecular Events from the

Biomedical Literature

Tiago Nunes, Sérgio Matos, José Luís Oliveira

TKG: A Graph-Based Approach to Extract Keywords from Tweets

Willyan Daniel Abilhoa, Leandro Nunes de Castro

\section{Image Processing, Tracking, Robotic, Control and Industrial Systems}

Outdoor Robotic Companion Based on a Google Android ${ }^{\mathrm{TM}}$ Smartphone and GPS Guidance

Eduard Clotet, Dani Martínez, Javier Moreno, Marcel Tresanchez,

Tomàs Pallejà, Davinia Font, Mercè Teixidó, Jordi Palacín

A Threshold Scheme for 3D Objects Based on Cellular Automata

Angel Martín del Rey

Generation Method of the Trigger Signal for the Automatic Capture

System to the Harmful Animals with Intelligent Image Processing

Fumiaki Takeda

2-Scene Comic Creating System Based on the Distribution of Picture

State Transition

Miki Ueno, Naoki Mori, Keinosuke Matsumoto

A Brief Approach to the Ear Recognition Process

Pedro Luis Galdámez, María Angélica González Arrieta,

Miguel Ramón Ramón 
Integration of Mobile Robot Navigation on a Control Kernel Middleware

Based System

Eduardo Munera Sánchez, Manuel Muñoz Alcobendas,

Juan Luis Posadas Yagüe, Jose-Luis Poza-Luján,

J. Francisco Blanes Noguera

Shared Map Convolutional Neural Networks for Real-Time Mobile

Image Recognition

William Raveane, María Angélica González Arrieta

\section{New Algorithms}

Using Multi-Objective Optimization to Design Parameters in

Electro-Discharge Machining by Wire

Alberto Ochoa, Lourdes Margain, Julio Arreola, Guadalupe Gutiérrez,

Geovani García, Fernando Maldonado

Learning Bayesian Networks Using Probability Vectors

Sho Fukuda, Takuya Yoshihiro

A Constraint Programming Approach to the Zahn's Decision Problem

Mhamdi Amel, Naanaa Wady

Multi-agent Model Based on Tabu Search for the Permutation Flow Shop

Scheduling Problem ... . . . . . . . . . . . . . . . . . . . . . . . . . . . . 519

Hafewa Bargaoui, Olfa Belkahla Driss

Neural-Based Method of Measuring Exchange-Rate Impact on

International Companies' Revenue

Svitlana Galeshchuk

Parallel Batch Pattern Training Algorithm for MLP with Two Hidden

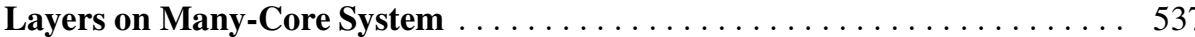

Volodymyr Turchenko

A Bee-Inspired Data Clustering Approach to Design RBF Neural

Network Classifiers

Dávila Patrícia Ferreira Cruz, Renato Dourado Maia,

Leandro Augusto da Silva, Leandro Nunes de Castro

An Item Influence-Centric Algorithm for Recommender Systems

Na Chang, Mhd Irvan, Takao Terano

Author Index 


\title{
Intelligent Lighting Control System
}

\author{
Elena García ${ }^{1}$, Sara Rodríguez ${ }^{1}$, Juan F. De Paz ${ }^{1}$, and Javier Bajo ${ }^{2}$ \\ ${ }^{1}$ Computer and Automation Department, University of Salamanca, Spain \\ ${ }^{2}$ Artificial Intelligence Department, Polytechnic University of Madrid, Spain \\ \{elegar,srg, fcofds\}@usal.es, jbajo@fi.upm.es
}

\begin{abstract}
This paper presents an adaptive architecture that allows centralized control of public lighting and intelligent management, in order to economise on lighting and maintain maximum comfort status of the illuminated areas. To carry out this management, architecture merges various techniques of artificial intelligence (AI) and statistics such as artificial neural networks (ANN), multiagent systems (MAS), EM algorithm, methods based on ANOVA and a Service Oriented Aproach (SOA). It performs optimization both energy consumption and economically from a modular architecture and fully adaptable to the current lighting systems possible. The architecture has been tested and validated successfully and continues its development today.
\end{abstract}

Keywords: Light sensors, intelligent systems, distributed systems, Autonomous control, Street lighting.

\section{Introduction}

Nowadays, the concept of Smart Cities is increasingly a common trend in technological projects. The balance with the environment and natural resources is a practical and responsible key for these paradigms, which aim to achieve a state of comfort for citizens and institutions based on sustainable development. In this respect, talk about energy efficiency is paramount, not only to reduce energy costs, but also to promote environmental and economic sustainability.

One of the main costs faced by councils in towns and cities is the lighting bill. According IDEA [1], throughout the 2010 in Spain there were about 4,800,000 points of light with an average power of $180 \mathrm{~W}$ and 4,200 hours of annual use. Representing an electricity consumption of 3,630 GWh / year, this is excessive consumption. The technological advances that are experiencing external lighting installations along with its intelligent use will allow reducing that as high consumption.

This research appears from a greater project by the research group of BISITE (Bioinformatics, Intelligent Systems and Technology Education) of the University of Salamanca, which is to build a system that allows centralized control street lighting as well as an intelligent management, in order to economize on illumination and maintain maximum comfort status of the illuminated areas. This is to avoid excessive illumination of areas, as there are many times that it is not necessary to maintain maximum light intensity for an optimal service. 
To validate the system in an experimental research level, a small test case is available, consisting of a portable installation of 5 luminaires with specific hardware. The functionality to be achieved is divided into two modules.

One module shall be responsible for the direct management of the various installations and control panels and will serve as a communication layer with each site, so as to allow as much control and monitoring in almost real-time of each facility and even luminaire. In this sense, the system must provide a service interface that can be accessed for each installation using a standardized interface independent of the underlying technology and hardware of each installation.

The other module, explained in this article, will have as its main objective the management of the lighting schedule for each installation, consumption management, and prediction. In this regard, a light planning is defined as light output level for each installation offered hourly. This light planning must be possible by programming user preferences, or by observing different decisive environment factors in determining the appropriate level of brightness for each site at each time. Thus, different factors come into play: astronomical clock, weather and traffic and pedestrian flow. Moreover, it will be interesting to make a prediction of consumption by the light patterns assigned to each zone, depending on its economic rate.

To carry out this management, the built system combines different statistics and artificial intelligence (AI) techniques such as artificial neural networks (ANN), multiagent systems (MAS), EM algorithm, methods based on ANOVA and a Service Oriented Approach (SOA) [5].

The article is structured as follows: Section 2 shows a state of the art concerning projects and research conducted in the field of Smart cities and light control, showing the most commonly used techniques in this field and carry out a comparison between them and the system presented. Section 3 shows the presented system, its operation and details of the techniques used. Section 4 describes the case study developed for system validation and finally, Section 5 some results and conclusions of this work.

\section{Background}

The concept of smart cities, smart environments, or smart homes [2] itself is still emerging in our society. Make a "smart" city is one of the objectives currently most often heard at the research as a strategy to mitigate the problems caused by the rapid growth of the urban population. Problems such as lack of resources, pollution, traffic congestion and deteriorating infrastructure are some of the many problems that increasingly large urban populations face [3].

One of the many definitions of Smart Cities is: "The use of smart computing technologies to make city services more intelligent, interconnected and efficient - which includes administration, education, health care, public safety, real estate, transportation and utilities."[4]. It seems clear that the purpose of these is sustainable economic development, based on new technologies (ICT) to provide better quality of life and prudent management of natural resources through the engagement of all citizens. 
Today, more and more cities around the world are committed to develop pilot projects related to this movement, some even in Spain, such as SmartSantander ${ }^{1}$ : for now the city has a great display of parking sensors to indicate to drivers the free sites. They also have a municipal Wi-Fi network that aims to cover the entire village, and even augmented reality applications to boost tourism. Málaga Smart City ${ }^{2}$ : the project aims at saving energy by micro power management: energy storage in batteries for use in buildings, street lighting and electrical transport, promoting the use of electric cars, etc. Smart City Valladolid-Palencia ${ }^{3}$ : considers two cities, adding transport between them as a problem and has smart meter network, integration of electric cars, energy efficiency in buildings, traffic organization, etc.

The current research works include the implementation and control of distributed lighting systems to facilitate the implementation of new infrastructure in a city or the optimization of existing infrastructure; further integration with other control systems and optimization of heating, cooling or controlling air quality. For instance, in [6] it presents a systematic approach to the modeling, optimization, control, and adaptation in a color-tunable LED lighting control system. Through light sensor feedback, the control system is able to achieve significant energy savings without substantially sacrificing lighting quality. The key techniques used here are an appropriate choice of cost function based on color metrics and the trade-off between quality of light and energy consumption for LED lighting systems. The authors in [7] employ formal methods for design a graph model, accompanied by means of control, including AI methods (rule-based systems, pattern matching) to design and control an outdoor lighting system. In this case, the work is focused only on the design phase and the control phase designing features such as dynamic, sensor-based control, multiple luminaire states and complex geometries. Other research on lighting control systems base their operation in image processing [8], fuzzy systems [9], cooperative methods and wireless sensor network (WSN) [10] or simulation algorithms [11] and predictive control [12] for energy optimization.

There are also some tools already developed as Lites ${ }^{4}$, that has temperature sensors, ambient light, power, motion detection; CityLight ${ }^{5}$, that allows remote management of lighting, fault detection and planning lighting patterns manually or Tvilight ${ }^{6}$ that regulates the lighting based on presence sensors and maintains minimum brightness in inactive hours.

\footnotetext{
${ }^{1}$ http: / / www. smartsantander.eu/

2 http://www. lacatedralonline.es/innova/system/Document/ attachments/12351/original/IDCCiudadesinteligentes.pdf

3 http: / / www.valladolidadelante.es/lang/modulo/ ?refbol=adelante-futuro\&refsec=smart-city-vyp\&idarticulo $=79302$

${ }^{4}$ http: / / www. lites-project. eu/lites-led-based-intelligentstreet-lighting-energy-saving

5 Teliko http://www.teliko.com/en/

${ }^{6}$ http://www.tvilight.com/
} 
This paper presents an adaptive architecture that allows centralized control of numerous public lighting installations. Specifically, it allows distributed and real-time intelligent control based on prediction and analysis techniques of lighting, one of the main shortcomings of the systems listed above. From a modular architecture fully adaptable to the current lighting systems possible, an energetic and economic optimization is possible. The architecture has been tested and validated successfully and continues its development today. The following sections describe the operation and technologies used in it and the results currently obtained.

\section{Proposed Architecture}

The system presented aims to frame the intelligent management of all public lighting, including monitoring and real-time control of the lights, and the establishment of lighting patterns that fit with the use of the public highway installations.

The following figure shows the context of the system, mainly composed of the control software (Intelligent Street Lighting Software) and the set of public-lighting installations, accessible via the internet. Facilities include special hardware for global and individual control of each luminary, while communication between devices is done by PLC. The control software is composed of three modules:

- The hardware abstraction layer allows communication with facilities regardless of the underlying hardware.

- The management server contains both device management and intelligent algorithms for efficient energy management. The "Data sources" module captures information related to pedestrian and traffic flow, weather data, and data about the monitoring of the facility. The "Data analysis" module deals with the study of information collected for the detection of foot traffic patterns, management of neural networks to predict consumption from light intensities, and estimates of consumption online. Finally, the "Luminosity patterns generator" module allows the creation of adequate light planning suitable for the specific facility lighting depending on the standards of pedestrian flow and weather conditions of each case.

- The web application provides access to all functionality for configuring lighting schedules, monitoring and control of facilities.

The ideal goal of the street lighting architecture design is that it can work well and provide safe and stable street lighting control for our daily life without human intervention. But human users should know whether the system is working normally or not. So the interaction between the system and human users is necessary. The system should also be controlled by human users manually in some particular situations. The system includes the ability to automatically interact ("smartly") or manually according to the lighting used and the needs of the specific case study. 


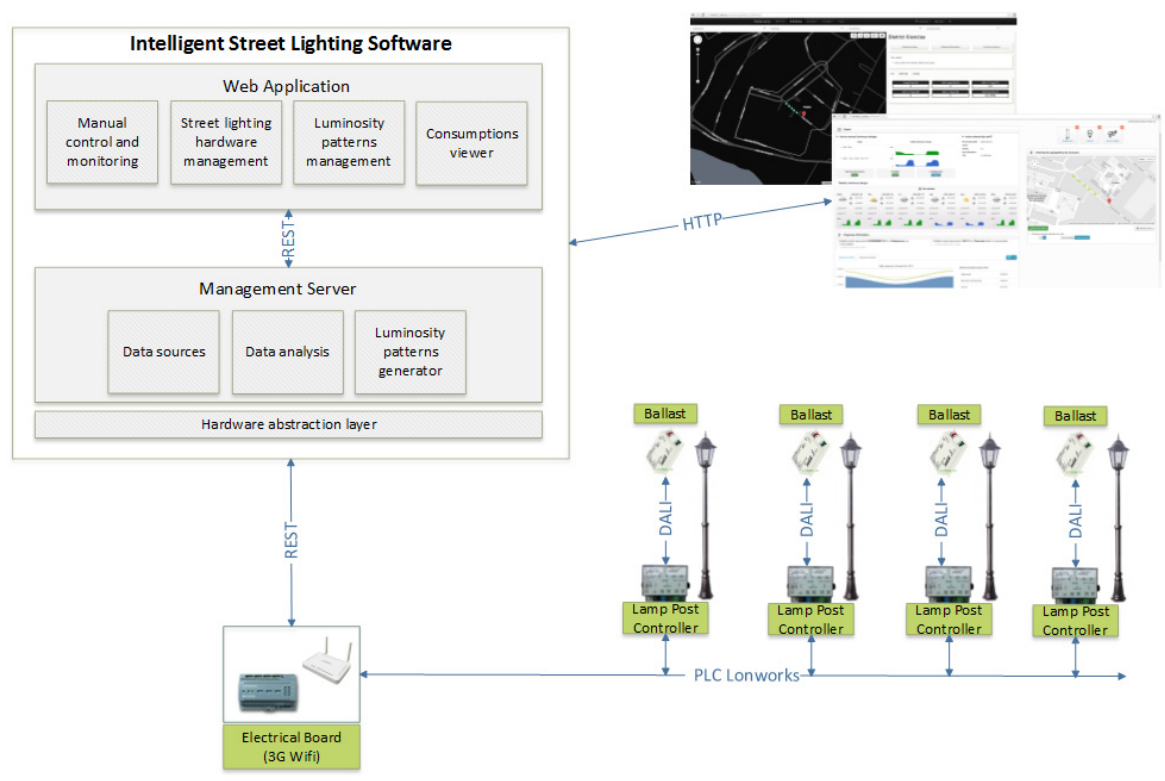

Fig. 1. Main system components

\subsection{Work Flow}

The establishment of adequate lighting configuration for each environment is one of the objectives of this project, meaning light configuration like a set of times at which the area is illuminated and the brightness levels associated with each time. This will save on lighting consumption, maintaining the state of maximum comfort in the lighted areas, as there are many occasions where it is not necessary to maintain a maximum level of light intensity to provide optimal service to the area, causing excessive consumption.

In the presented system the light designs can be set in a manual or smart way. In the first, the user is encouraged to plan the time slots (in hours) and the luminous flux of each time slot. In the second way, we proceed to the observation of different environmental factors that may be influential in determining adequate lighting for the particular area, such as flow or pedestrian traffic, or weather conditions each time, which influence the level of ambient light, especially near the hours of sunrise and sunset times.

The flow diagram of Figure 2 shows the procedures to complete the light patterns depending on ambient factors and the different user preferences. It is possible to observe, with common parts, two different workflows, which correspond to the process of generating light patterns for a given period of time. One of the flows can generate patterns without establishing a maximum estimated expenditure, and the other, setting it. Maximum expenditure means the maximum amount to spend on lighting bill for the period over which the light patterns are concluded. 


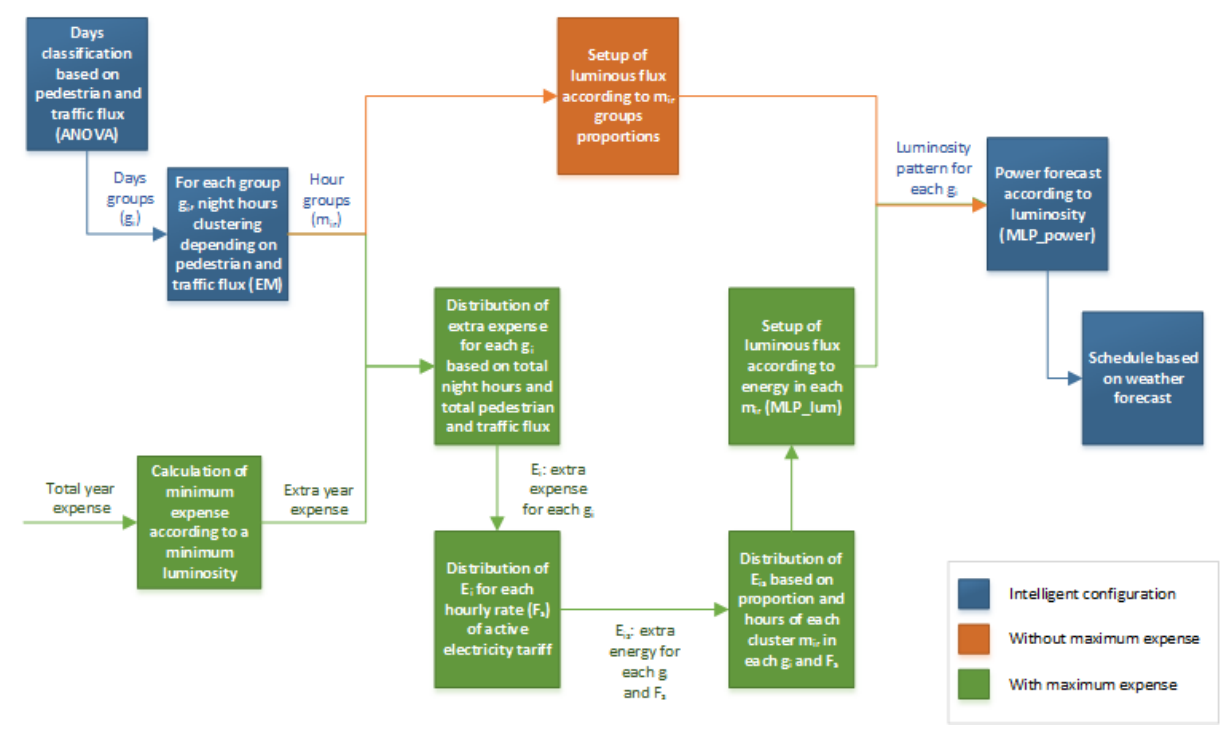

Fig. 2. Workflow procedures followed for lighting

Both processes share the initial logic. As a first step, historical data of pedestrian and traffic flow of several weeks are taken. Subsequently, a classification is performed by analysis of variance (ANOVA) [13] to determine what day of the week patterns share pedestrian and traffic flow according to the different hours of the period.

The periods (days) that share the same characteristics, according to this analysis, also will share luminous pattern. To determine the set of similar days is necessary to take into account different variables such as day of the week, time of day and the volume of people / traffic that is located in that time slot and day of the week. Not being quantitative variables is necessary to apply cluster techniques such as ANOVA to draw similarity between groups. Here is to be applied two-way ANOVA with repetition. The factors are the day of the week and the time slot, the time slot is considered factor group.

After obtaining the groups of days, for each group is applied a clustering algorithm (Expectation-maximization EM) [14][15] to determine at what time of night usually spend a similar number of pedestrians or traffic. These clusters of hours generated for each group of days will result in different light levels appropriate to pedestrian or traffic flow.

After these two steps, the flowchart shows a bifurcation. The left branch corresponds to the process to follow if you do not set a maximum expenditure, which mainly follows a simple process of adjusting lighting levels based on the proportion of generated clusters after EM technique for each group of days. Moreover, the right branch serves logic followed when a maximum flow is established. The steps followed in this last branch focus primarily on optimal distribution of the amount with which it has to provide the same light levels in scenarios with similar environmental characteristics. At all times a minimum configurable brightness is guaranteed, because in order to comply with the appropriate legislation, the area will not be left with insufficient lighting although the amount entered by the user is less than the cost of this. The distribution amount is performed based on features such as hourly rate, with 
or without time restrictions, the evening hours that can affect and the proportion of clusters based on traffic and pedestrian flows.

The two branches obtain different light patterns depending on the groups of days generated with the ANOVA technique. The penultimate step in the flowchart is shared and consists of the prediction of the spending of the lighting design completed, which in the case of the branch with maximum expenditure, will coincide with a small margin of error depending on the expenditure estimation technique used. The estimate of expenditure is performed by a neural network MLP (Multi-Layer Perceptron) [16][17] that predicts power level in function of lumens and is trained with historical data of the luminaire type used in each installation.

The other shared step in the workflow, optional for users, is a replanning that is performed periodically to adjust light patterns established previously to climatic conditions. This process consists in checking the prediction of the weather to advance or delay the time on and off lights in the hours of dawn and sunset. This is for that the lighting design conforms to the lighting conditions of the place in which the system is installed. In this way, for any day in which bad weather (rain, fog, etc.) is expected, the system will turn off the luminaries sooner or later that the usual hours, coinciding with the hours of dawn and sunset each day. This process is repeated weekly, so that the light patterns are sent weekly to the control node of the area.

\subsection{Distribution of Expenditure}

To calculate the distribution of the maximum $E_{T}$ in $Z$ time entered by the user is first necessary to calculate the minimum amount of expenditure $E_{\min }$ to a minimum brightness $L_{\text {min }}$. This time period has $N h$ overnight hours. One MLP network is used to predict the spending power of the luminaires used depending on the required level of brightness. The additional expenditure $E$ will be distributed for generating light patterns.

$$
\begin{gathered}
L_{\text {min }} \rightarrow R N A_{\text {pow }} \rightarrow \text { Pow }_{\text {min }} \\
E_{\text {min }}=\text { Pow }_{\text {min }} N h \\
E=E_{T}-E_{\text {min }}
\end{gathered}
$$

The first step taken is to distribute the amount $E$ between groups of days gi generated in the ANOVA process. This distribution is based on the number of hours of night $N h i$ of each group $i$ and pedestrian and traffic flow $P_{i}$ that exists in that group.

The calculation of traffic and pedestrian flow $P_{i}$ of each $g_{i}$ is done by average people who go through every night $\overline{P d_{l}}$ and the number of days $D_{i}$ that belongs to $g_{i}$, taken the average of the historical data used before (Fig 1). The number of people is limited, and is equal to the upper bound in case of exceeding this bound.

$$
\begin{gathered}
P_{i}=\overline{P d_{\iota}} D_{i} \\
\overline{P d_{\iota}}=\frac{1}{J K} \sum_{i=1}^{I} \sum_{j=1}^{J} \sum_{k=1}^{K} y_{i j k} / \mathrm{j} \in \mathrm{g}_{i}
\end{gathered}
$$

Both $N h$ and $\mathrm{P}$ variables can have different degrees of influence $\rho$ at the time of allocation of the amount $E$. The extra expense of each group gi is given by equation (3). 


$$
E_{i}=\frac{N h_{i}}{N h} E \rho_{N h}+\frac{P_{i}}{P} E \rho_{P}
$$

Where:

$$
\begin{gathered}
N h=\sum_{i=1}^{I} N h_{i} ; P=\sum_{i=1}^{I} P_{i} \\
0 \leq \rho_{N h} \leq 1 ; 0 \leq \rho_{P} \leq 1 ; \quad \rho_{N h}+\rho_{P}=1
\end{gathered}
$$

Once the distribution of expenditure for each group is done, $E_{i}$, a process recurs distribution thereof, in this case between different times $F s$ of stating the electricity tariff associated with the area to be illuminated. To do this, we take into account the proportion $\left(w_{i r}\right)$ and time of use in hours $\left(n_{i r}\right)$ in each group $m_{i r}$ (which represents the values that are classified in the $E M_{r}$ cluster of each group $g_{i}$ ANOVA), and the price of the energy in each time slot, $L_{s}$.

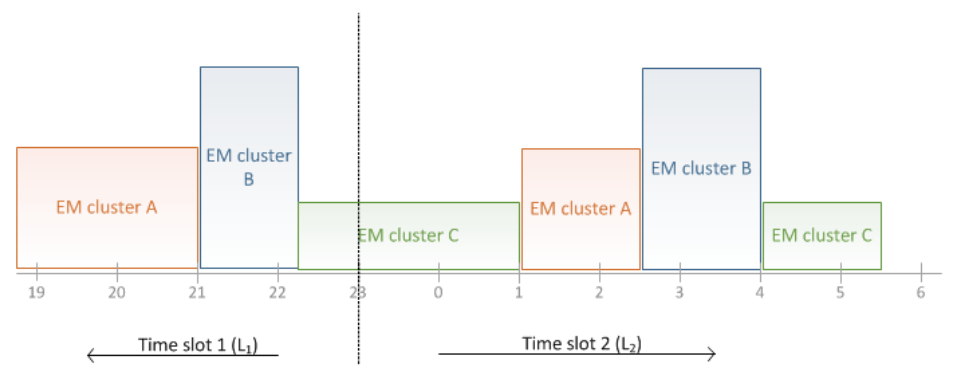

Fig. 3. Area of time slots

The figure above shows graphically a possible deployment scenario clusters $m_{i r}$ result of the EM algorithm, where each cluster is represented by a rectangle. The $\mathrm{x}$ axis represents time in hours, while the y-axis represents the proportion of clusters $\left(w_{i r}\right)$, which is determined by the average of pedestrian and traffic flow determined for each group $m_{i r}$. The distribution of expenditure $E_{i}$ is done by calculating the total area of each time slot $F_{s}$ weighted price of energy in these slots. Thus, a fair distribution of the expenditure is insured for, thus able to illuminate with the same light flow spaces schedules with similar environmental factors (3).

$$
\begin{gathered}
E_{i s}=\left(\sum_{r=1}^{R_{i}} n_{i r} w_{i r}\right) L_{s} x \\
E_{i}=\sum_{s=1}^{S} E_{i s} \\
w_{i r}=\frac{\sum_{i=1}^{I} \sum_{j=1}^{J} \sum_{k=1}^{K} y_{i j k}}{I J K} /_{\mathrm{j} \in \mathrm{g}_{i}, \mathrm{i} \in \mathrm{m}_{i r}}
\end{gathered}
$$


Once the distribution of expenditure for each time slot $F_{s}$ of each group of days $g_{i}$ is made, the price of energy does not vary for each slot of the group. In this step, each expendture $E_{i s}$ becomes equivalent to the energy consumed in the slot $s$ of the days that belong to the group $i$ during the period of time $Z$, that the user chose at the beginning of the process. The distribution of extra power (it will continue denoting as $E_{i s}$ ) for each hour $h \in \mathrm{H}_{\text {irs }}$ (set of hours that belong to $F_{s}$ slot grouped in subgroup $m_{i r}$ hours, of the initial group $g_{i}$ ) was performed similarly to the previous step manner, to continue to ensure a fair distribution. It will consider both the size of cluster $w_{i r}$ as the number of hours used $n_{i r}$.

$$
\begin{aligned}
& p_{h}=w_{i r} x / h \in H_{i r s} \\
& E_{i s}=\left(\sum_{r=1}^{R_{i}} n_{i r} w_{i r}\right) x
\end{aligned}
$$

The expression (6) denotes $p_{h}$ as the extra power to supply each hour $h \in \mathrm{H}_{\text {irs. }}$ In this way, the power to supply in each hour to all luminaries $\left(p_{t h}\right)$ will be the minimum power (1) of each hour more extra power calculated $p_{h}$.

$$
p_{t h}=\frac{P o w_{\min }}{N h}+p_{h}
$$

At this point, the power supply to each luminaire in every hour (simply dividing $p_{t h}$ by the number of luminaires) is known. For the resulting light output of the power supplied a MLP network that predicts light output from input power is utilized. The equivalence between luminous flux and power tends to be linear, so this approximation is quite accurate: in most cases, if you double the power is spent, it will get twice the luminosity. However, the use of MLP networks to predict luminosity based on power or vice versa, is performed for the sections in which it is not linear: each model of luminaire can light to a minimum power; moreover the expenditure of each luminaire can be influenced by the facility in which it is (because it is necessary more hardware, etc.). Thus, the system achieves more accurately approximate the luminosity that is going to have with a certain power. Or conversely, the cost of having the lights burning with a certain luminous flux. Knowledge of the luminous flux which has every hour for each group $g_{i}$ presupposes already done the lighting pattern to be followed for each group of days. Whereupon, planning lighting design is approximated to the expenditure that the user wants to spend and to the pedestrian and traffic flow patterns in the area, depending of the desired degree of influence set.

\section{Case Study}

To develop the first prototype a hardware solution installed in a briefcase was purchased. Fig. 4 shows a photo of the prototyping environment that emulates an installation of five street lamps with a control node. Four lamps interior and one exterior 
were used to test the control system while maintaining low cost hardware. Each lamp is controlled by an adjustable ballast that is behind it, hidden in the top panel. A luminaire controller regulates and monitors each ballast and liaises with the control node.

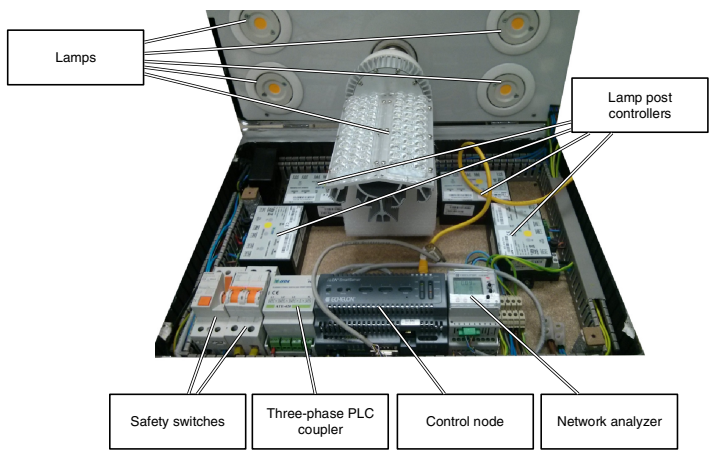

Fig. 4. Prototyping environment that simulates an installation of five streetlights

Luminaire controllers (ISDE brand ASL-510-TCH) are placed inside each lamp and communicate via PLC with the control node, but also can be placed at the beginning of a line of street lamps. These controllers interpret commands received through the line to regulate the output of the ballasts of the lamps using the DALI protocol. Also they monitor the status of the lamps, the instantaneous consumption and power supply of each lamppost.

The chosen control node is an Echelon Smart Server, a general purpose controller for automation of non-critical processes. In street lighting systems, is able to control and monitor up to 192 single or double lamps head through PLC. It offers a SOAP interface for configuration and remote management that has been used for integration with the developed system. The PLC signal injected by the control node replicates in three phases using a phase coupler [three-phase coupler PLC]. The network analyzer is the CVM-MINI brand model Circutor. It connects via a parallel port RS-485 to Echelon SmartServer, with which it communicates using the MODBUS protocol. For the prototype system for estimating pedestrian flow IP camera TP-LINK DSC-942L interior placed on a window was used.

\section{$5 \quad$ Results, Conclusions and Future Work}

Figure 5 shows some of the results obtained by using the system. The upper panels show data of pedestrian flow in two consecutive weeks (one week in purple and the other blue). After applying the analysis of variance traffic patterns are detected and rated day is done, resulting in two groups: green, weekdays, and blue, on weekends. The lower graphs show the generated light designs for each group of days. Using the EM algorithm, hours with similar traffic are detected, adjusting a luminosity level in each group. 


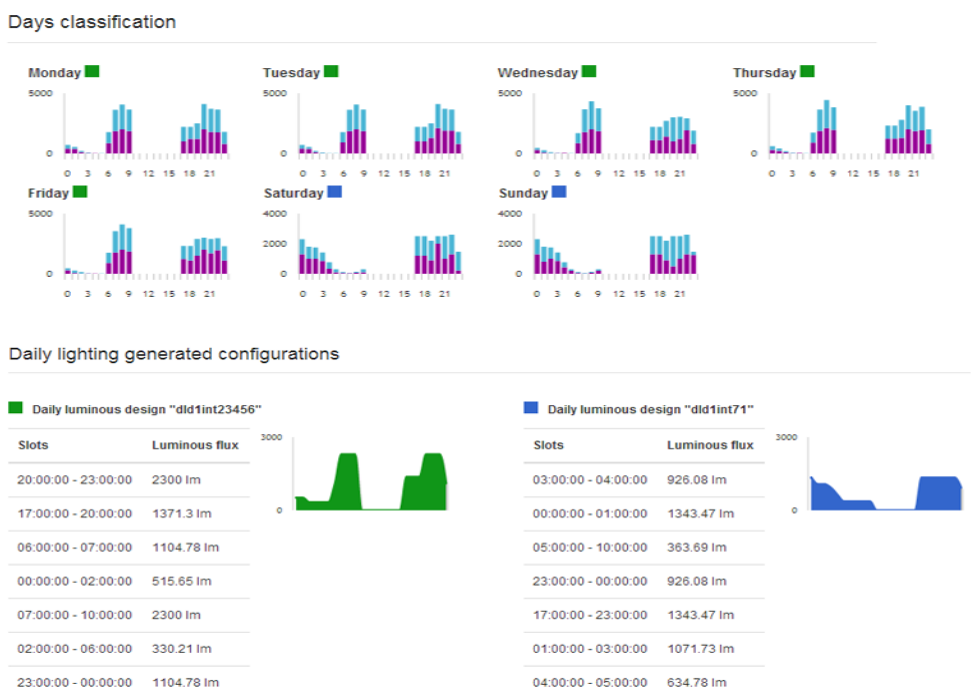

Fig. 5. Results

Figure 6 shows a prediction of both daily consumption and annual calendar designed. The green line represents the expense of having all lights full brightness during nighttime hours. The blue area represents the estimated model to the application of consumption. In this case, an approximate savings of $25 \%$ is achieved while maintaining the maximum light intensity at peak traffic and pedestrian flow.

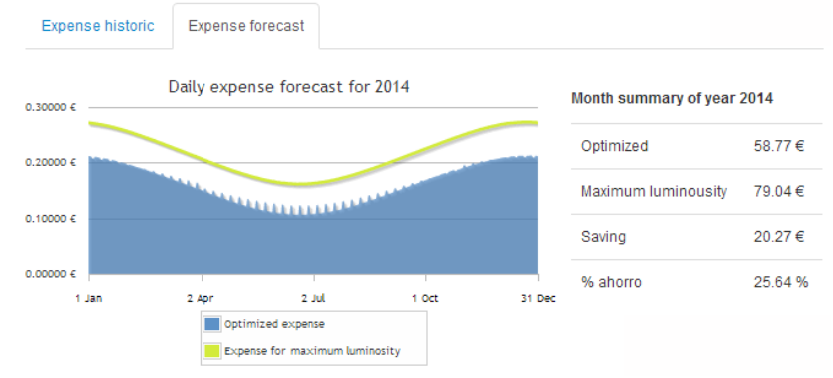

Fig. 6. Prediction of daily and annual consumption

The system is able to set lighting schedules all public lighting installations controlled and can say that this measure contributes to energy savings achieved by regulating the light intensity. The user can define their own light patterns, where lighting pattern is the hours in which the lights are on and at what level of brightness. Likewise, it is possible to assign different light patterns each day.

Furthermore, not only the lighting schedule can be established according to the user preferences, but the system has the necessary mechanisms to automatically adjust light levels based on traffic or pedestrian flow provided for each zone. To achieve 
this, the system is based on historical information of people flow and makes a clasification of days to find patterns of pedestrian/traffic flow. Based on these patterns, the system establishes an appropriate lighting design for each type of day. In this sense, we have applied intelligent techniques and algorithms (ANOVA, EM clustering technique, MLP) correctly and a process that fuses all together for the conclusion of the lighting schedule is made. In addition, a distribution algorithm that reduces spending and complies with the minimum luminosity and brightness levels at all times is presented. Finally, the application also allows the user to query historical data related to the luminance calendars that have been established on site, and the historical use of them.

In conclusion say that it is very difficult to find prototypes that are based on historical data of pedestrian and traffic flow to adjust the luminosity of the areas. The systems are often reactive, not predictive. The main reason for developing the system is based on the prediction of pedestrian / traffic flow is the savings in hardware. Place a camera in the area for pedestrians and vehicles spot for a while, is much cheaper than having every few luminaires a presence sensor that regulates the brightness depending on the passage of pedestrians and vehicles, in addition to the constant change light intensity emitted by the luminaires could punish excessively. Future work will focus on the following three aspects. (1) Add other sensors to the lamp member and investigate how to use sensor fusion to further improve intelligence level of the system. (2) Develop a system of alerts that happen in the real-time hardware: cast a light, overvoltage on the line, etc. (3) Develop new algorithms to make the lamp members cooperate with each other.

Acknowledgements. This work has been carried out by the project PIRSES-GA2012-318878.

\section{References}

[1] IDAE, Instituto para la Diversificacion y Ahorro de la Energía, http : / / www. idae.es / (last access January 12, 2014)

[2] Gómez-Romero, J., Serrano, M.A., Patricio, M.A., García, J., Molina, J.M.: Contextbased Scene Recognition from Visual Data in Smart Homes: An Information Fusion approach. ACM/Springer Journal of Personal and Ubiquitous Computing. Special Issue on Sensor-driven Computing and Applications for Ambient Intelligence 16(7), 835-857 (2012)

[3] Chourabi, H., Nam, T., Walker, S., Gil-Garcia, J.R., Mellouli, S., Nahon, K., Pardo, T.A., Scholl, H.J.: Understanding Smart Cities: An Integrative Framework. In: 45th Hawaii International Conference on System Sciences, pp. 2289-2297 (2012)

[4] Washburn, D., Sindhu, U., Balaouras, S., Dines, R.A., Hayes, N., Nelson, L.E.: Helping CIOs Understand "Smart City" Initiatives. Growth 17 (2009),

http://c3328005.r5.cf0.rackcdn.com/

73efa931-0fac-4e28-ae77-8e58ebf74aa6.pdf

[5] Daigneau, R.: Service Design Patterns: Fundamental Design Solutions for SOAP/WSDL and RESTful Web Services, 1st edn. Addison-Wesley Professional (2013) 
[6] Afshari, S., Mishra, S., Julius, A., Lizarralde, F., Wason, J.D., Wen, J.T.: Modeling and control of color tunable lighting systems. Energy and Buildings 68(Pt. A), 242-253 (2014)

[7] Wojnicki, I., Ernst, S., Kotulski, L., Se dziwy, A.: Advanced street lighting control. Expert Systems with Applications 41(4 Pt. 1), 999-1005 (2014)

[8] Carrillo, C., Diaz-Dorado, E., Cidrás, J., Bouza-Pregal, A., Falcón, P., Fernández, A., Álvarez-Sánchez, A.: Lighting control system based on digital camera for energy saving in shop windows. Energy and Buildings 59, 143-151 (2013)

[9] Yang, C., Fan, S., Wang, Z., Li, W.: Application of fuzzy control method in a tunnel lighting system. Mathematical and Computer Modelling 54(3-4), 931-937 (2011)

[10] Zhang, J., Qiao, G., Song, G., Sun, H., Ge, J.: Group decision making based autonomous control system for street lighting. Measurement 46(1), 108-116 (2013)

[11] Bourgeois, D., Reinhart, C., Macdonald, I.: Adding advanced behavioural models in whole building energy simulation: A study on the total energy impact of manual and automated lighting control. Energy and Buildings 38(7), 814-823 (2006)

[12] Barra, K., Rahem, D.: Predictive direct power control for photovoltaic grid connected system: An approach based on multilevel converters. Energy Conversion and Management 78, 825-834 (2014)

[13] Miller Jr., R.G.: Beyond ANOVA: basics of applied statistics. CRC Press (1997)

[14] Dempster, A.P., Laird, N.M., Rubin, D.B.: Maximum Likelihood from Incomplete Data Via the EM Algorithm. Journal of the Royal Statistical 39, 1-38 (1977)

[15] Little, R., Rubin, D.: Statistical Analysis with Missing Data. Wiley \& Son, New York (2002)

[16] Haykin, S.: Neural Networks: A Comprehensive Foundation, 2nd edn. Prentice Hall (1998) ISBN 0-13-273350-1

[17] Collobert, R., Bengio, S.: Links between Perceptrons, MLPs and SVMs. In: Proc. Int'1 Conf. on Machine Learning, ICML (2004) 